\title{
RETRACTION NOTE
}

\section{"Sorsby Fundus Dystrophy-Related Mutation} in Tissue Inhibitor of Metalloproteinases-3 Impairs Regulation of Its Expression in Mouse Fibroblasts" by G. T. Sukhikh and G. M. Soboleva,

Vol. 143, No. 1, pp. 64-67, January 2007

The above article has been withdrawn because the incomplete work was published without the approval of the team where the work took place. 